\title{
Bayesian Case-Exclusion and Personalized Explanations for Sustainable Dairy Farming (Extended Abstract)*
}

\author{
Eoin M. Kenny ${ }^{1,2,4 \dagger}$, Elodie Ruelle ${ }^{3,4}$, Anne Geoghegan ${ }^{3}$, Laurence Shalloo ${ }^{3,4}$, Micheál \\ O'Leary $^{3}$, Michael O'Donovan ${ }^{3}$, Mohammed Temraz ${ }^{1,2,4}$ and Mark T. Keane ${ }^{1,2,4}$ \\ ${ }^{1}$ University College Dublin, Dublin, Ireland \\ ${ }^{2}$ Insight Centre for Data Analytics, UCD, Dublin, Ireland \\ ${ }^{3}$ Teagasc, Moorepark. Co. Cork, Ireland \\ ${ }^{4}$ VistaMilk SFI Research Centre \\ \{eoin.kenny, mohammed.temraz, mark.keane\}@insight-centre.org, \\ \{elodie.ruelle, anne.geoghegan, laurence.shalloo, michael.oleary, michael.odonovan\}@teagasc.ie
}

\begin{abstract}
Smart agriculture (SmartAg) has emerged as a rich domain for AI-driven decision support systems (DSS); however, it is often challenged by useradoption issues. This paper reports a case-based reasoning (CBR) system, PBI-CBR, that predicts grass growth for dairy farmers, combining predictive accuracy and explanations to improve user adoption. PBI-CBR's novelty lies in the use of Bayesian methods for case-base maintenance in a regression domain. Experiments report the tradeoff between predictive accuracy and explanatory capability for variants of PBI-CBR, and how updating Bayesian priors each year improves performance.
\end{abstract}

\section{Introduction}

Although the promise of artificial intelligence (AI) in SmartAg is usually advertised as increasing productivity, in the future it may be more about improving sustainability [Gafsi et al., 2006; Lindblom et al., 2017]. As climate change accelerates, AI's main future contribution may be more about helping farmers to measure, profile, and predict the outcomes of farm-management decisions in ways that mitigate their environmental impacts. However, this future depends on the development of AI-enabled, decision-support systems (DSS) that are both predictively accurate and explainable to the end user. Here, an existing DSS, called PastureBase Ireland (PBI), is enhanced using case-based reasoning (CBR) techniques, in the so-called PBI-CBR system. This new DSS predicts grass growth for dairy farmers and offers explanations designed to improve user adoption. PBICBR's key technical novelty is its use of Bayesian CaseExclusion, to "clean up" user-entered data; a technique that by excludes outlier cases from the prediction process using prior beliefs about data distribution(s), thus reducing error and improving explanations. In the remainder of this intro-

\footnotetext{
* Originally Publication [Kenny et al., 2019] at ICCBR 2019.

${ }^{\dagger}$ Contact Author.
}

duction, the sustainability context for this work is briefly described, before outlining the structure of the paper.

\subsection{Context: Agriculture, Sustainability and AI}

Concerns about the impact of agriculture on climate change and the development of sustainable models are growing [Lindblom et al., 2017]. The agricultural sector and consumers are faced with opposing views from climate change denial, to proposals that animal agriculture is responsible for $18-51 \%$ of greenhouse grass emissions [Steinfeld et al., 2006; Goodland et al., 2009]. However, a recent middle ground has emerged pushing for a quick move to sustainable farming systems [Poux and Aubert, 2018]; the so-called agroecology perspective. For example, in the dairy sector, agroecology proposes a move to pasture-based systems, where animals are predominantly fed on grass outdoors rather than on meal and supplements indoors. This pasture-based system has the potential to be sustainable if there is better grass management (e.g., using grass as a carbon sink). However, these innovations depend on precision technologies, using AI, for monitoring variables such as the climate and grass growth.

\subsection{PastureBase Ireland (PBI) \& Dairy Farming}

SmartAg often depends upon providing new DSSs for farmers to aid them in making complex decisions about how to manage their farms, balancing productivity and sustainability [Lindblom et al., 2017]. These systems need to be predictively accurate, easy to use (and interpretable), and they need to be able to support actionable decision-making in the face of increasing climate disruption. The present work enhances the existing PBI DSS used for grass-fed, pasture-based dairy farming systems in Ireland.

Since 2013, Ireland's national agricultural research organization, Teagasc, have provided PBI to support Irish dairy farmers (6,000+ users). Among other features, the PBI database has weekly records of grass covers for individual farms (2013-present). A farm's "grass cover" is the amount of grass available on that farm for cows to eat. PBI-CBR uses historical grass-cover data to predict grass growth rates on a farm from one week to the next. These calculations allow farmers to budget feed for their herds, to determine if there 
is sufficient (inexpensive) grass available or whether (expensive) meal needs to be bought, meal that incurs additional carbon costs as it tends to be imported (e.g., from the USA).

We used the PBI dataset recorded from thousands of private farms in Ireland between 2013-2017. The primary feature of concern is the average grass growth rate for a farm since the last grass cover recorded, but location features (Farm ID-anonymized and County) are also important for explanation purposes. To explain its predictions PBI-CBR aims to provide explanatory cases from the same farm, or failing that, a case from a nearby farm in the same county.

\subsection{Outline of Paper}

Section 2 discusses noise in the PBI dataset and how a Bayesian approach is both useful and intuitive for the problem. Section 3 describes Expt. 1 which compares four systems on accuracy and explanatory success. Section 4 describes Expt. 2 which shows how updating priors using Bayesian techniques can improve prediction accuracy and may well help to deal with disruptive climate events. Section 5 reviews related work before making some final conclusions.

\section{Noise: A Tale of Two Datasets}

The grass-growth domain faces many of the typical problems that arise in SmartAg; notably, that the data is noisy, in part, because it has been entered by end-users (who are, often, non-technical). The PBI dataset has growth-data entered by farmer end-users, data that is known to contain errors, miss-recordings, and subjective estimates. For example, some grass-growth recordings are based on physical measurements with specialized devices (i.e., plate meters), whereas others are based on visual inspection. This noise in the dataset is dealt with by removing outlier cases, using a novel method, called Bayesian case-exclusion; this method uses a separate gold-standard dataset, gathered under controlled conditions (which is noise-free), to "clean up" the farmer-entered data, to create what we call the working-farm dataset.

\subsection{The Gold Standard Dataset}

The gold-standard dataset of grass-growth measurements we used, covers 28 years of carefully-controlled, weekly measurements in which samples, taken by researchers from the same pasture, were cut, dried, and weighted on a weekly basis at the Teagasc Moorepark Dairy Research Centre, Fermoy, Co. Cork. These measurements are idealized, but very accurate and can thus serve as a good benchmark for determining outlier cases in the PBI dataset, which we exclude using our Bayesian case-exclusion method.

\subsection{PBI Dataset}

The PBI dataset, used to construct the working-farm casebase, came from the weekly grass-covers entered by farmers in PBI; these grass-covers are farmer's estimates of grass available on a given farm on a given day and were used to make the grass-growth predictions for one week ahead. Some of these records are known to be in-error; for example, often multiple entries are made on the same day, where the last entry of the day was the intended record. For the years

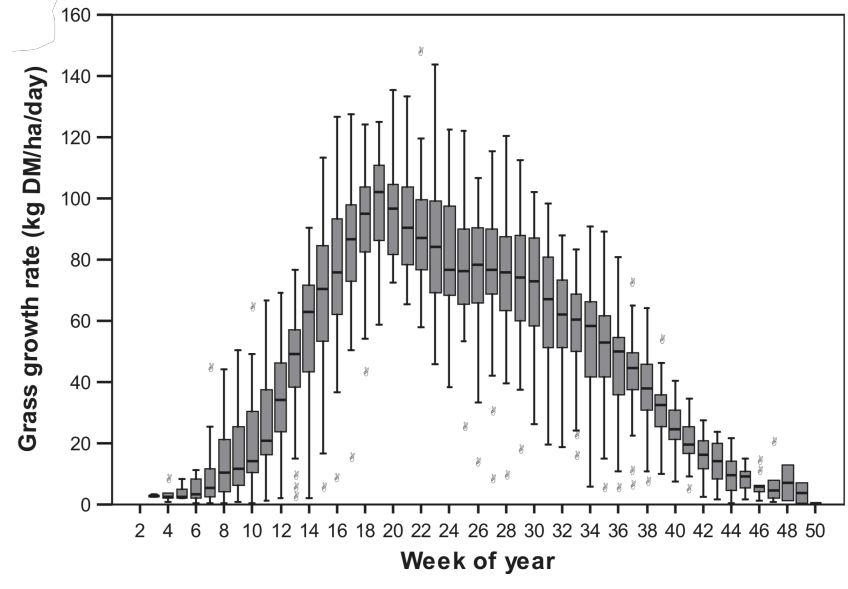

Figure 1: The gold-standard dataset of grass growth measurements from 1982-2010 at Teagasc, Animal and Grassland Research and Innovation Centre, Moorepark, Fermoy, Co. Cork, Ireland [HurtadoUria et al., 2013], where the distribution of grass growth each week of the year is given as box plots.

2013-2017, this dataset had 99,087 grass-cover records, that reduced to 92,635 when same-day entries were removed.

Case generation. Let a farm's data be $f=$ $\left\{x_{1}, x_{2}, \ldots x_{n}\right\}$, where $x_{i}$ is a grass cover for a day, and $n$ the total number of covers (in chronological order). The features of $x_{i}$ used to generate a case $\left(C_{i}\right)$ are the average growth rate since the previous grass cover ( $g r)$, the week $(w k)$, month $(m t h)$, and season (seas) in which the grass cover was recorded. Weather data $\left(w_{i}\right)$ at the county level was scraped from Met Éireann, and added as an average from $x_{i}$ until $x_{i+1}$. The weather information in $w_{i}$ is the maximum daily temperature (maxt), the average soil temperature $10 \mathrm{~cm}$ below the surface (soilt) on a given day, and the average global radiation ( $\mathrm{grad}$ ) on a given day. Finally, $g r$ from $x_{i+1}$ was added to $C_{i}$ as the target feature. Thus, a case is represented as:

$$
\begin{array}{r}
C_{i}\left(x_{i}, w_{i}, x_{i+1}\right)=\left\langle x_{i}(\text { gr }, w k, \text { mth }, \text { seas }),\right. \\
\left.w_{i}(\text { maxt }, \text { soilt }, \text { grad }), x_{i+1}(\text { gr })\right\rangle
\end{array}
$$

Case base construction. Taking the raw-data, the cases as defined in Equation 1 were constructed; however, given that the system has to predict one week ahead, only those cases where the target $x_{i+1}(g r)$ was recorded 5-9 days after $x_{i}$ were included in the case base. Also, cases from January and December were excluded (as they tend to show zero growth). Finally, only those cases with accurate historical weather information until the next grass cover were considered (weather is crucial for predicting grass growth). These steps resulted in a working-farm case-base of 20,760 cases.

\subsection{The Current Experiments}

In the remainder of this section, two experiments are reported that test several variants of the Bayesian case-exclusion idea. In Expt. 1, we examine what happens in this predictive CBRsystem when cases are not excluded (Control), versus experimental systems in which they are. In Expt. 2, we explore 
adaptive Bayesian case-exclusion, and consider the data as a time series where priors derived from the gold-standard distribution are updated year-on-year.

\section{Experiment 1: Bayesian Case-Exclusion}

In this domain an error of $\leq 10 \mathrm{~kg} \mathrm{DM} / \mathrm{ha}$ is sufficient, but the major problem is uncertainty in the working-farm case-base, hence we use Bayesian case-exclusion to exclude likely outlier cases when making predictions. PBI-CBR also explains predictions by referencing nearest neighbouring cases from the same farm or county used in the prediction. The four variants of the system tested were:

- Control. A basic system that uses all the cases in the working-farm case base.

- Exclude-2sd. A Bayesian system that excludes cases two-standard deviations away from the weekly, mean growth-rates of the gold-standard dataset (see Fig. 1). The rationale being that grass growth in a given week approximates a normal distribution. Formally, the data for growth rate $(G R)$ in a given week across all years in the gold-standard dataset approximates $G R \sim N\left(\mu, \sigma^{2}\right)$, where $N$ is a normal distribution with parameters $\mu$ and $\sigma$ for the mean and standard deviation, respectively. All cases outside $\mu \pm 2 \sigma$ are excluded. This step reduces the working-farm case-base by $42 \%(\mathrm{~N}=12,042$ cases).

- Exclude-3sd. This is identical to the Exclude-2sd system but $\mu \pm 3 \sigma$ is used to exclude cases. This reduces the working-farm case-base by $21 \%(\mathrm{~N}=16,443$ cases $)$.

- Transform-3sd. This is a Bayesian system that transforms the growth-rates of cases using the gold-standard distribution. That is, the distribution of growth in a given week from the gold-standard dataset [ $\left.G R \sim N\left(\mu, \sigma^{2}\right)\right]$ is used to transform the growth-rate values of cases for the same week in the working-farm case-base, to fit to the parameters $\mu$ and $\sigma^{2}$. Formally, to transform the growth-rate $(g r)$ in a grass cover $x$ in any given week of the year:

$$
y_{g r}=\left(x_{g r}-\mu\right) \frac{\sigma_{p}}{\sigma}+\mu_{p}
$$

where $x_{g r}$ is the growth rate in grass cover $x, y_{g r}$ is the transformed growth rate of $x_{g r}, \mu$ and $\sigma$ are the mean and standard deviation for the overall growth rate in that week in the working-farm case-base, respectively, and $\mu_{p}$ and $\sigma_{p}$ are the mean and standard deviation for the overall growth rate in that week in the gold-standard dataset, respectively. The intuition being that the goldstandard dataset is closer to the ground-truth. Note, in this system cases that fall outside $\mu_{p} \pm 3 \sigma_{p}$ after the transformation are still excluded, so the working-farm case-base is reduced by $2 \%(\mathrm{~N}=20,282$ cases $)$.

As we shall see, exclusion methods improve prediction accuracy, with varying levels of explanatory success. The transform system retains as many cases as possible, aiding accuracy and explanatory success. Interestingly, there are indications that the transformed case-base is closer to the ground truth as the correlation of Pearson's $r$ between $\operatorname{grad}$ and $G R$ across all cases increases from $r=0.53$ to $r=0.66$ after transformation, reflecting known dependencies between such radiation and grass-growth [Ruelle et al., 2018].

\subsection{Method: Procedure and Measures}

For each system variant Monte Carlo cross-validation was used with 30 re-sampling iterations, each time taking 80/20\% data for training and testing, respectively. A standard $k$-NN algorithm was used for case retrieval and prediction. Selected values of $k$ ranging from 5-1000 were tested for each system variant. For each evaluation of $k$ in each system, three measures were taken: (i) the mean absolute error (MAE) (ii) the \%Farm-Explanation-Success (\%FES; i.e., the percentage of times the $k$-nearest-neighbours contained a case from the same farm as the query), and (iii) the \%County-ExplanationSuccess (\%CES; i.e., the percentage of times the $k$-nearestneighbours contained a case from the same county).

\subsection{Results and Discussion}

Fig. 2a shows the results the system variants for all values of $k$ in three graphs, one for each measure: MAE, \%FES, and $\%$ CES. Overall, MAE is worst for the lowest $k$ with some improvement in at $k=20-35$. Regarding \%FES all systems are similar, though success does change for different values of $k$. For all systems \%FES is poor for low values of $k$, but beyond $k=50$ it rises to $80 \%$; showing that higher values of $k$ have enough cases from the same farm to explain the predictions. For all systems, \%CES starts high $(\sim 80 \%)$ and rapidly reaches $\sim 100 \%$.

Overall, the Control system never gets lower than an MAE of 15. Similarly, from the two exclusion-systems only

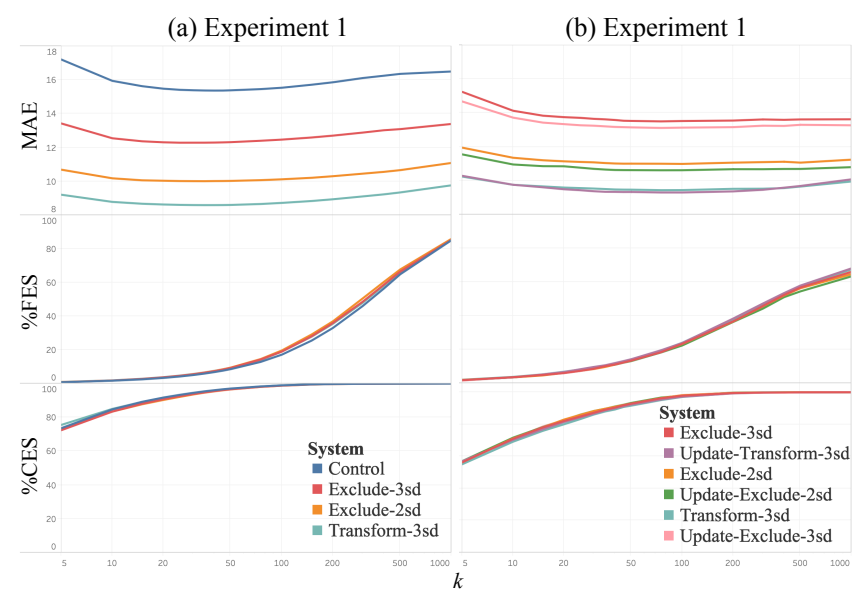

Figure 2: The tradeoff between error and explanation. (a) Expt. 1 shows that as the value for $k$ approaches 1000 , more explanatory cases are retrieved, but the MAE for all systems also increases. Transform-3sd has the best MAE of $\sim 8.6 \mathrm{~kg} \mathrm{DM} / \mathrm{ha} /$ day at $k \sim 35$, but same-farm explanatory success is low at $\sim 7 \%$; however, at $k=1000$, the tradeoff is balanced, with the MAE still acceptable and $\%$ FES at $85 \%$. (b) Expt. 2 shows MAE is improved for almost every update-variant, although the improvement in the transform-system is minimal; explanatory success and MAE are similar to Expt. 1, but poorer, likely due to less training data. Finally, note the log scale on the $\mathrm{x}$-axis. 
Exclude-2sd with $k=35$ has the somewhat acceptable MAE of $\sim 10.01 \mathrm{~kg} \mathrm{DM} / \mathrm{ha} /$ day. Overall, the Transform-3sd system is the best with a MAE $<10 \mathrm{~kg} \mathrm{DM} /$ ha/day for all values of $k$; here it is capable of delivering county explanations very consistently, but more data would be needed for similar success in farm explanation-retrieval.

\section{Experiment 2: Updating Priors}

In Expt. 1 Bayesian exclusion/transformation of cases resulted in improved performance. However, all of these systems exclude cases using static parameters from the goldstandard dataset. In recent years, climate change is impacting the distributions of grass growth, and Expt. 2 tries to rectify this by updating Bayesian priors year-on-year. So, Expt. 2 has six versions of PBI-CBR, three systems tested in Expt. 1, and three variants of these systems in which priors were updated (Update-Exclude-2sd, Update-Exclude-3sd, Update-Transform-3sd). The procedure is described next.

\subsection{Updating Bayesian Priors}

To perform the updating, priors are taken from the goldstandard dataset and then progressively used on each year's data from the PBI-dataset to update them; the idea being to try to track shifts in climate over time. First, take the goldstandard dataset and, binning all its data into weeks, for any given week, let the growth rate $(G R)$ approximate a normal distribution $G R \sim N\left(\mu, \sigma^{2}\right)$, where $\mu$ and $\sigma^{2}$ are its mean and variance, respectively. In 2013, all the data for this week was processed into cases (see Section 4.2). Then, we proceed with transformation or exclusion methods on these cases depending on the system variant (using $\mu$ and $\sigma^{2}$ as in Expt. 1), which gives the new data $D=\left\{C_{1}, C_{2} \ldots C_{n}\right\}$, where $n$ is the number of cases. Take the prior to be $\mu \sim N\left(\mu_{0}, \sigma_{0}^{2}\right)$, where the value $\sigma_{0}$ is initially chosen as 4 , and $\mu_{0}$ is initially chosen as $\mu$. Here the value for $\sigma^{2}$ is assumed to remain fixed. Bayes rule shows the posterior (for a given week) is proportional to the likelihood times the prior, in addition, because $\sigma^{2}$ and $\sigma_{0}^{2}$ are known we can ignore the constant of proportionality and derive that the posterior $\mu_{p}$ is:

$$
\mu_{p} \sim N\left(\frac{\sigma^{2}}{\sigma^{2}+\sigma_{0}^{2} n} \mu_{0}+\frac{\sigma_{0}^{2}}{\sigma^{2}+\sigma_{0}^{2} n} n \bar{x}, \frac{\sigma^{2} \sigma_{0}^{2}}{\sigma^{2}+\sigma_{0}^{2} n}\right)
$$

where $\bar{x}$ is the empirical mean of the growth rates in the cases of $D$, for a full derivation see [Murphy, 2007].

Using Equation 3 we update values for $\mu_{0}$ and $\sigma_{0}^{2}$, the new value of $\mu_{0}$ was then used to update the original $\mu$ from the gold-standard dataset, which was used with $\sigma^{2}$ (the fixed variance from the gold-standard dataset) to repeat the whole process in 2014 for the same week. This process is repeated for all weeks of each year until the end of 2016 when all training data was collected. The latest priors in each week were again used to exclude or transform cases in 2017 for evaluation.

\subsection{Method: Procedure and Measures}

For each system the case base was split in a 60/40\% ratio for training and testing, respectively; the former being the PBI data from 2013-2016 and the latter 2017. Crucially, these results will be different from identical systems in Expt. 1 because of the different splits (there is also less training data here). For case retrieval, a standard $k-\mathrm{NN}$ was again used with selected values for $k$ ranging from 5-1000. The three measures used were again MAE, \%FES, and \%CES.

\subsection{Results and Discussion}

Fig. 2b shows the results, that generally replicate Expt. 1. Regarding MAE, as before the transformation-versions do better than the exclusion-versions, with $k=75$ being optimal for all systems. Expt. 2 shows that Bayesian updating improves all systems at nearly every value of $k$. Regarding explanation the overall curve-shapes are similar to Expt. 1, with maximum values being $\% \mathrm{FES}=68 \%$ and $\% \mathrm{CES}=100 \%$, in contrast to $\% \mathrm{FES}=85.94 \%$ and $\% \mathrm{CES}=99.98 \%$ in Expt. 1 . Acceptable tradeoffs for accuracy and explanation are achieved for both of the transform systems in that at $k=1000$ the MAE is $\sim 9.95 \mathrm{~kg} \mathrm{DM} / \mathrm{ha} /$ day with $\sim 67.5 \%$ explanatory-success rate for same-farm cases in both systems.

\section{Related Work}

Case base maintenance is a notable area of research in CBR [Smiti and Elouedi, 2011]. However, most methods have focused on classification [Hart, 1968; Gates, 1972; Ritter et al., 1975; Guan et al., 2009; Aha et al., 1991; Markovitch and Scott, 1988], as opposed to regression [Redmond and Highley, 2010]. Redmond and Highley [2010] did try to convert Edited Nearest Neighbors [Wilson, 1972] for regression by assigning two hyperparameters, but they acknowledge that applying the classification algorithms to regression is difficult. Our method requires no hyperparameters, though it does require the specification of a prior(s).

XAI within CBR has been shown to be important in intelligent systems [Kenny and Keane, 2019; Keane and Kenny, 2019], with some consideration of CBR recommenders in SmartAg [Cho et al., 2012]. Frameworks have been proposed for explanation in CBR XAI [Sørmo et al., 2005], but there are few CBR-applications in SmartAg (for one exception see Branting et al.'s [2001] work on grasshopper-infestation).

\section{Conclusion}

We have shown that a CBR system can be used for a DSS in dairy farming to predict grass growth and provide personalized case-based explanations. To deal with noise in the data, we have introduced Bayesian case-exclusion to use prior knowledge to identify and exclude noisy data. Furthermore, we have shown that Bayesian calculations for updating priors year-on-year also improves performance [Kenny et al., 2019]. These systems have the ability to improve the sustainability of dairy farming in the future. Our more recent research [Temraz et al., 2020] has shown that these techniques can continue to deliver accurate predictions in the face of climate change by using the previously-excluded "outlier" cases in later years (such as the hot summer of 2018). Hopefully, though we may experience significant climate shifts, there will always be a case somewhere in the historical record that can provide accurate predictions, and by extension explanations. 


\section{References}

[Aha et al., 1991] David W Aha, Dennis Kibler, and Marc K Albert. Instance-based learning algorithms. Machine learning, 6(1):37-66, 1991.

[Branting et al., 2001] Karl Branting, John D Hastings, and Jeffrey A Lockwood. CARMA: A case-based range management advisor. In $I A A I$, pages 3-10, 2001.

[Cho et al., 2012] Yongyun Cho, Kyoungryong Cho, Changsun Shin, Jangwoo Park, and Eun-Ser Lee. A recommend service based on expert knowledge model in agricultural environments. In International Conference on Hybrid Information Technology, pages 189-194. Springer, 2012.

[Gafsi et al., 2006] Mohamed Gafsi, Bruno Legagneux, Genevieve Nguyen, and Patrice Robin. Towards sustainable farming systems: Effectiveness and deficiency of the french procedure of sustainable agriculture. Agricultural Systems, 90(1-3):226-242, 2006.

[Gates, 1972] Geoffrey Gates. The reduced nearest neighbor rule (corresp.). IEEE transactions on information theory, 18(3):431-433, 1972.

[Goodland et al., 2009] Robert Goodland, Jeff Anhang, et al. Livestock and climate change: What if the key actors in climate change are... cows, pigs, and chickens? World Watch Magazine, 2009.

[Guan et al., 2009] Donghai Guan, Weiwei Yuan, YoungKoo Lee, and Sungyoung Lee. Nearest neighbor editing aided by unlabeled data. Information Sciences, 179(13):2273-2282, 2009.

[Hart, 1968] Peter Hart. The condensed nearest neighbor rule (corresp.). IEEE transactions on information theory, 14(3):515-516, 1968.

[Hurtado-Uria et al., 2013] Cristina Hurtado-Uria, Deirdre Hennessy, Laurence Shalloo, Declan O'Connor, and Luc Delaby. Relationships between meteorological data and grass growth over time in the south of ireland. Irish Geography, 46(3):175-201, 2013.

[Keane and Kenny, 2019] Mark T Keane and Eoin M Kenny. How case-based reasoning explains neural networks: A theoretical analysis of XAI using post-hoc explanation-byexample from a survey of ANN-CBR twin-systems. In International Conference on Case-Based Reasoning, pages 155-171. Springer, 2019.

[Kenny and Keane, 2019] Eoin M Kenny and Mark T Keane. Twin-systems to explain artificial neural networks using case-based reasoning: Comparative tests of featureweighting methods in ANN-CBR twins for XAI. In Twenty-Eighth International Joint Conferences on Artifical Intelligence (IJCAI), Macao, 10-16 August 2019, pages 2708-2715, 2019.

[Kenny et al., 2019] Eoin M Kenny, Elodie Ruelle, Anne Geoghegan, Laurence Shalloo, Micheál O'Leary, Michael O'Donovan, and Mark $\mathrm{T}$ Keane. Predicting grass growth for sustainable dairy farming: A CBR system using bayesian case-exclusion and post-hoc, personalized explanation-by-example (XAI). In International Conference on Case-Based Reasoning, pages 172-187. Springer, 2019.

[Lindblom et al., 2017] Jessica Lindblom, Christina Lundström, Magnus Ljung, and Anders Jonsson. Promoting sustainable intensification in precision agriculture: review of decision support systems development and strategies. Precision Agriculture, 18(3):309-331, 2017.

[Markovitch and Scott, 1988] Shaul Markovitch and Paul D Scott. The role of forgetting in learning. In Machine Learning Proceedings 1988, pages 459-465. Elsevier, 1988.

[Murphy, 2007] Kevin P Murphy. Conjugate bayesian analysis of the gaussian distribution. $\operatorname{def}, 1(2 \sigma 2): 16,2007$.

[Poux and Aubert, 2018] Xavier Poux and Pierre-Marie Aubert. An agroecological europe in 2050: multifunctional agriculture for healthy eating. 2018.

[Redmond and Highley, 2010] Michael A Redmond and Timothy Highley. Empirical analysis of case-editing approaches for numeric prediction. In Innovations in Computing Sciences and Software Engineering, pages 79-84. Springer, 2010.

[Ritter et al., 1975] G Ritter, H Woodruff, S Lowry, and $\mathrm{T}$ Isenhour. An algorithm for a selective nearest neighbor decision rule (corresp.). IEEE Transactions on Information Theory, 21(6):665-669, 1975.

[Ruelle et al., 2018] E Ruelle, D Hennessy, and L Delaby. Development of the moorepark st gilles grass growth model (most gg model): A predictive model for grass growth for pasture based systems. European journal of agronomy, 99:80-91, 2018.

[Smiti and Elouedi, 2011] Abir Smiti and Zied Elouedi. Overview ofmaintenance for case based reasoning systems. International Journal of Computer Applications, 975:8887, 2011.

[Steinfeld et al., 2006] Henning Steinfeld, Pierre Gerber, TD Wassenaar, Vincent Castel, Mauricio Rosales, Mauricio Rosales, and Cees de Haan. Livestock's long shadow: environmental issues and options. Food \& Agriculture Org., 2006.

[Sørmo et al., 2005] Frode Sørmo, Jörg Cassens, and Agnar Aamodt. Explanation in Case-Based Reasoning:Perspectives and goals. Artificial Intelligence Review, 24(2):109-143, October 2005.

[Temraz et al., 2020] Mohammed Temraz, Eoin M. Kenny, Elodie Ruelle, Laurence Shalloo, and Mark T. Keane. Precision agriculture in a time of climate change: Using CBR to predict grass growth for a sustainable agriculture facing disruptive climate events. Insight Tech. Report., 2020.

[Wilson, 1972] Dennis L Wilson. Asymptotic properties of nearest neighbor rules using edited data. IEEE Transactions on Systems, Man, and Cybernetics, (3):408-421, 1972. 